\title{
Feasibility of intraoperative diagnosis of lung adenocarcinoma in situ to avoid excessive resection
}

\author{
Toshiyuki Shima ${ }^{1,2}$, Tomonari Kinoshita ${ }^{1,2}$, Naomichi Sasaki ${ }^{1}$, Mao Uematsu ${ }^{1}$, Yusuke Sugita ${ }^{1}$, \\ Reiko Shimizu ${ }^{1}$, Masahiko Harada ${ }^{1}$, Tsunekazu Hishima ${ }^{3}$, Aya Yamamoto ${ }^{4}$, Hirotoshi Horio ${ }^{1}$
}

${ }^{1}$ Department of Thoracic Surgery, Tokyo Metropolitan Cancer and Infectious Diseases Center Komagome Hospital, Tokyo, Japan; ${ }^{2}$ Division of Thoracic Surgery, Keio University School of Medicine, Tokyo, Japan; ${ }^{3}$ Department of Pathology, Tokyo Metropolitan Cancer and Infectious Diseases Center Komagome Hospital, Tokyo, Japan; ${ }^{4}$ Department of Radiology, Tokyo Metropolitan Cancer and Infectious Diseases Center Komagome Hospital, Tokyo, Japan

Contributions: (I) Conception and design: T Kinoshita; (II) Administrative support: M Harada, T Hishima, A Yamamoto; (III) Provision of study materials or patients: T Kinoshita, M Harada, T Hishima, A Yamamoto, H Horio; (IV) Collection and assembly of data: T Shima, N Sasaki, M Uematsu, Y Sugita, R Shimizu, M Harada, T Hishima, A Yamamoto; (V) Data analysis and interpretation: T Kinoshita, H Horio; (VI) Manuscript writing: All authors; (VII) Final approval of manuscript: All authors.

Correspondence to: Tomonari Kinoshita. Department of Thoracic Surgery, Tokyo Metropolitan Cancer and Infectious Diseases Center Komagome Hospital, 3-18-22 Honkomagome, Bunkyo-ku, Tokyo 113-8677, Japan. Email: kinotomo0415@gmail.com.

Background: Limited lung resection is generally believed to be available for lung adenocarcinoma in situ (AIS). At our institute, intraoperative hematoxylin-eosin staining of frozen-section slides is routinely performed for evaluating tumor invasiveness after partial resection to avoid excessive lung resection. This study aimed to evaluate the feasibility and usefulness of intraoperative frozen-section diagnosis of AIS.

Methods: We retrospectively reviewed 143 patients with 151 AISs diagnosed by intraoperative frozen sections between 2012 and 2019 at our institute. All patients underwent limited resection because of the result of intraoperative frozen-section diagnosis.

Results: The total concordance rate between the diagnoses of AIS by intraoperative frozen sections and postoperative paraffin-embedded sections was $82.7 \%$ for 151 nodules. Although 21 minimally invasive adenocarcinomas (MIA) and 5 invasive adenocarcinomas were diagnosed as AIS intraoperatively, no patient had tumor recurrence after resection. Among 125 pathologically proven cases of AIS postoperatively, there were 67 (53.6\%) radiologically invasive tumors including ground-glass nodules (GGNs) with part-solid component or pure-solid nodules.

Conclusions: This intraoperative evaluation of frozen-section slides will help surgeons avoid excessive lung resection for AIS that was radiologically diagnosed as invasive adenocarcinoma. Intraoperative frozen-section diagnosis will provide to be clinically useful and lead to less invasive surgical treatment for lung nodules.

Keywords: Intraoperative frozen-section diagnosis; adenocarcinoma in situ (AIS); lung cancer; limited resection

Submitted Aug 21, 2020. Accepted for publication Dec 03, 2020.

doi: $10.21037 /$ jtd-20-2710

View this article at: http://dx.doi.org/10.21037/jtd-20-2710

\section{Introduction}

Because of the prevalence of high-resolution computed tomography (HRCT), patients with small and resectable non-small cell lung cancer (NSCLC) are increasing in number worldwide (1-4). Lung nodules with ground-glass opacity (GGO), so-called ground-glass nodules (GGNs), are also frequently detected by computed tomography (CT), which indicates less invasive lung adenocarcinoma. Indeed, these nodules are mostly diagnosed as lung adenocarcinoma including invasive adenocarcinoma, minimally invasive adenocarcinoma (MIA), and adenocarcinoma in situ 


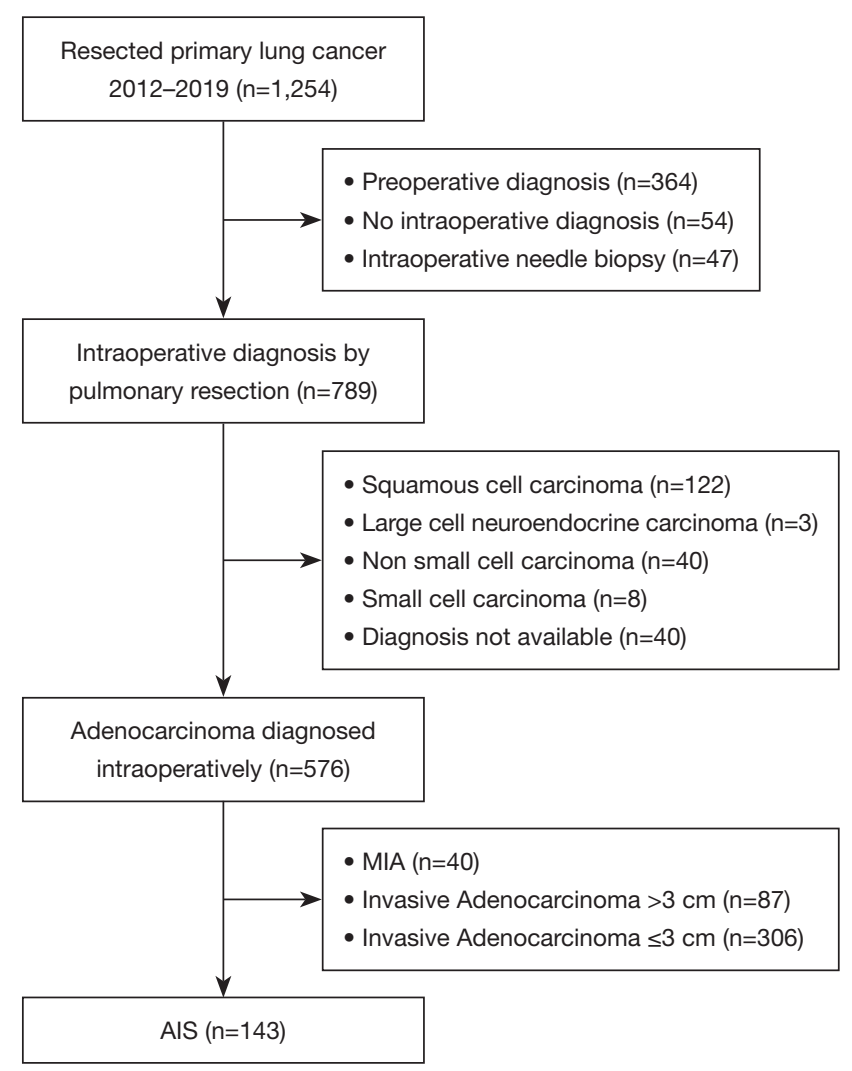

Figure 1 A diagram of inclusion and exclusion process. Between 2012 and 2019, 1,257 patients with lung cancer who underwent curative resection were identified. After exclusion, 147 patients were included in the analysis. AIS, adenocarcinoma in situ.

(AIS) pathologically (5). Some ongoing clinical trials are investigating the clinical feasibility of limited lung resection for MIA and AIS (6). Although the definitive consensus to indicate the safety of limited resection for less or noninvasive lung adenocarcinomas has not been confirmed, limited resection for AIS is very safe and feasible because of the lack of invasiveness. In 2016, the tumor, node, and metastasis (TNM) classification of lung cancer was revised in the eighth edition, based on a huge number of patient data $(7,8)$. The revision consisted of major changes in the $\mathrm{T}$ descriptors, focusing on the invasive component size of the tumor. Lung nodules diagnosed as "Tis" without lymph node and distant metastasis are classified as stage 0 .

In surgical cases, discordance between the radiological and pathological diagnosis for GGN often occurs. Although a GGN with a solid part more than $5 \mathrm{~mm}$ is suspected as invasive adenocarcinoma, which requires standard resection (lobectomy) based on preoperative HRCT findings, the final diagnosis is confirmed as AIS pathologically. For such cases, limited resection of the lung including wedge resection and segmentectomy would be enough to completely control tumor growth including recurrence. Some reliable intraoperative methods to show the invasiveness of lung tumors, especially for part-solid GGNs, are needed to avoid excessive resection of the lung.

This study aimed to evaluate the feasibility and usefulness of intraoperative frozen-section diagnosis of AIS.

We present the following article in accordance with the STARD reporting checklist (available at http://dx.doi. org/10.21037/jtd-20-2710).

\section{Methods}

\section{Patients}

Of the 1,253 patients who underwent curative resection for lung cancer at our institute between January 2012 and December 2019, we retrospectively reviewed patients diagnosed as having AIS intraoperatively (iAIS) by comparing to HRCT findings and referring to the final diagnosis using formalin-fixed and paraffin-embedded (FFPE) specimens. Exclusion criteria are listed in Figure 1. Because limited resection for MIA and IA is not established as a feasible operation yet, cases with MIA and invasive adenocarcinoma diagnosed intraoperatively were excluded.

\section{Extent of surgical resection}

The type of lung resection determined the surgical technique. In all cases, wedge resections were performed by 3 -port video-assisted thoracic surgery (VATS), and segmentectomy and lobectomy were performed by open thoracotomy. We found the targeted nodule by palpation intraoperatively and performed partial resection with a surgically safe margin. When the nodule was not palpable or identified, we performed segmentectomy or lobectomy depending on preoperative CT findings including the tumor size and tumor location. On the other hand, we performed CT-guided lipiodol marking before partial resection for some non-palpable nodules to identify their position during surgery. The non-palpable nodule was defined as a small diameter $(<1 \mathrm{~cm})$, deep $(>1 \mathrm{~cm}$ from visceral pleura), or pure-ground glass density on preoperative CT findings. When the resected nodule was diagnosed as AIS based on frozen sections, as described below, and the cutend was confirmed to be cancer-free pathologically, we 
closed the incision without additional lung resection and lymphadenectomy. If invasive adenocarcinoma or another histological tumor was confirmed, we performed lobectomy and lymph node dissection. We explained these procedures of limited resection to each patient with a solitary nodule, and we received his/her acceptance preoperatively.

\section{Radiological evaluation}

Without prior knowledge of the pathological diagnoses, the chest radiologists (A.H.) and her colleagues visually classified tumors into 3 subgroups: pure GGN, part-solid GGN, and pure-solid nodule. Moreover, part-solid GGNs were classified into the following 2 patterns according to the solid component size (SS): $\leq 5$ and $>5 \mathrm{~mm}$. GGN was defined as an area of slight homogenous increase in density that did not obscure the underlying vascular markings. Solid part was defined as an area of increased opacity that completely obscured underlying vascular markings. Partsolid was defined as nodules containing both GGN and solid. The radiologists measured the longest diameter of each tumor and the maximum diameter of the solid part of each tumor using electronic calipers.

\section{Pathological evaluation}

Regarding frozen-section diagnosis, after sectioning the frozen samples, some slides of tumor center were stained with hematoxylin-eosin (H.E.). For final diagnosis, after fixing the specimens with $10 \%$ formalin and embedding them in paraffin, staining was performed with H.E. and elastica Van Gieson to assess the degree of pleural and vascular invasions.

According to the fourth edition of the World Health Organization histological classification, the cases were reviewed and staged according to the eighth edition of the TNM classification of the Union for International Cancer Control (8). Briefly, AIS was defined as a localized, small $(\leq 3 \mathrm{~cm})$ adenocarcinoma with growth restricted to neoplastic cells along with pre-existing alveolar structures (lepidic growth) that lacks stromal, vascular, or pleural invasion; MIA was defined as a small, solitary adenocarcinoma $(\leq 3 \mathrm{~cm})$ with a predominantly lepidic growth pattern and $\leq 5 \mathrm{~mm}$ invasion in the greatest dimension of anyone foci. AIS or MIA was not diagnosed if any of the following conditions were present: (I) histological subtypes other than a lepidic growth pattern (i.e., alveolar, papillary, micropapillary, and/or solid) or (II) if the tumor invaded lymphatics, blood vessels or pleura, or contained necrosis.

Three or more pathologists (T.H. and his colleagues) performed the histological evaluation without knowing preoperative clinical data. Disagreements among observers on the invasion size were discussed using a multiheaded microscope until agreement. The disagreements were mainly due to discrepancies on whether the interstitial fibrotic hypertrophy accompanied the destruction of preexisting alveolar structures.

When an intraoperatively diagnosed AIS tumor was confirmed as invasive adenocarcinoma postoperatively, we strongly recommended that the patients undergo additional lung resection after the operation.

\section{Statistical analyses}

All patients received every 3 months follow-ups at our outpatient department for the first 2 years post-resection, and every 6months for the third year, and once per year for the subsequent year, consisting of physical examination, blood analysis, chest radiography, and routine CT scans of the chest and upper abdomen. Brain magnetic resonance imaging and radionuclide bone scan were performed every year. Positron emission tomography of the neck, chest, and abdomen was performed when patients with suspicious symptoms or recurrence signs. Tumor recurrence was determined according to the results of these evaluations. Overall survival (OS) was measured from the date of pulmonary resection to the date of death from any cause or the date on which the patient was last known to be alive. Recurrence-free survival (RFS) was measured as the interval between the date of resection and the date of recurrence, date of death from any cause, or the date on which the patient was last known to be alive and recurrence-free (as confirmed by the last CT performed before death). Survival curves were plotted according to the Kaplan-Meier method and compared using the log-rank test. Data were analyzed with the statistical Standard Package for Social Sciences software (SPSS, standard version 25.0; IBM Corp., Armonk, NY, USA).

\section{Ethical statement}

The study was conducted in accordance with the Declaration of Helsinki (as revised in 2013). This study was approved by the Institutional Review Board (IRB) of Tokyo Metropolitan Cancer and Infectious Diseases Center Komagome Hospital (IRB number 2481). The need 
for written informed consent was waived because of the retrospective study design.

\section{Results}

\section{Patient characteristics}

Between 2012 and 2019, 143 patients were diagnosed as having iAIS by frozen section. None had received preoperative chemotherapy or thoracic radiotherapy. In 12 of 151 cases, we performed lobectomy regardless of frozensection diagnosis because the tumor was located close to the hilum (Table 1).

\section{A typical case in which we could avoid excessive lobectomy}

Images of a typical case that we could avoid excessive lobectomy, including HRCT and stained slides, are shown in Figure 2. Although this nodule has no ground-glass component radiologically, the frozen-section slide shows that all the tumor cells are well-differentiated without the destruction of the original fibrotic texture, consistent with AIS's typical findings. In this case, as aforementioned, we finished the operation without further resection. The final pathology diagnosis was AIS.

\section{The concordance of diagnosis between frozen-section and FFPE slides}

Among 151 iAIS cases, 125 nodules were confirmed as AIS by FFPE-based diagnosis (accuracy rate, $82.7 \%$ ). The other cases consisted of 21 MIAs and 5 invasive adenocarcinomas. For patients with proven MIA nodules, we decided to follow up with them closely in our outpatient clinic because the need for additional resection in such cases is not generally confirmed. The surgical and pathological characteristics of 5 cases with proven invasive adenocarcinoma are shown in Table 2. Case 1, 2 and 3 underwent lobectomy due to a location close to the hilum. Case 4 underwent segmentectomy due to a keep safety margin. Case 5 underwent wedge resection. In these cases, as aforementioned, we strongly recommended additional lobectomy, and we performed a second surgical resection. The HRCT and pathology slides of 2 cases among such 5 cases are shown in Figure 3. Although frozen-section slides did not reveal invasive features, such as destruction of the original fibrotic texture, FFPE slides show invasive components, such as papillary and acinar patterns, indicating invasive adenocarcinoma.

The factor for frozen-section errors included infiltration of the lesion observed in deeper paraffin-embedded sections because the pathologist saved the true maximum diameter slide section level for final diagnosis. For reference, we present the breakdown list of intraoperative and final diagnosis of intraoperatively-diagnosed $\leq 3 \mathrm{~cm}$ adenocarcinomas (Table 3).

\section{Cases in which excessive resection was avoided because of frozen-section diagnosis}

Among 125 pathologically proven AIS cases postoperatively, there were $67(53.6 \%)$ radiologically invasive tumors including part-solid GGNs or pure-solid nodules. Without these frozen-section diagnoses, we would have performed excessive resection for lung nodules (Table 4).

\section{Postoperative survival}

Among 143 patients, 5 patients died of another disease during the observation period. No patient had tumor recurrence postoperatively. The respective 5-year OS rates were $94.3 \%$ in AIS cases, 94.4\% in MIA, and 100\% in invasive adenocarcinoma (Figure 4). RFS rates were the same as OS rates because of no recurrence. There was no statistical difference in postoperative survival between AIS cases, MIA, and invasive adenocarcinoma.

\section{Discussion}

We achieved a high avoidance rate of excessive lung resection for patients with pathologically proven AIS. In the current TNM staging of lung cancer, particularly in T staging, the size of the invasive component is important (8). Clinically, we frequently encounter patients with partsolid GGN because CT is prevalently performed. Some studies have demonstrated that not the total tumor size but the degree of invasion in such cases is very important information because the extent of invasion reflects its invasiveness, which is significantly associated with postoperative prognosis (9). Although bronchioalveolar carcinoma was formerly diagnosed as adenocarcinoma, a GGN smaller than $3 \mathrm{~cm}$ in diameter without an invasive component is diagnosed as stage 0 currently. A couple reports have revealed that limited resection of the lung could be enough for a non-invasive GGN to control recurrence when an adequate surgical margin is confirmed (10). 
Table 1 Clinical, radiological, surgical, and pathological characteristics of 143 patients with intraoperatively-diagnosed adenocarcinoma in-situ (iAIS)

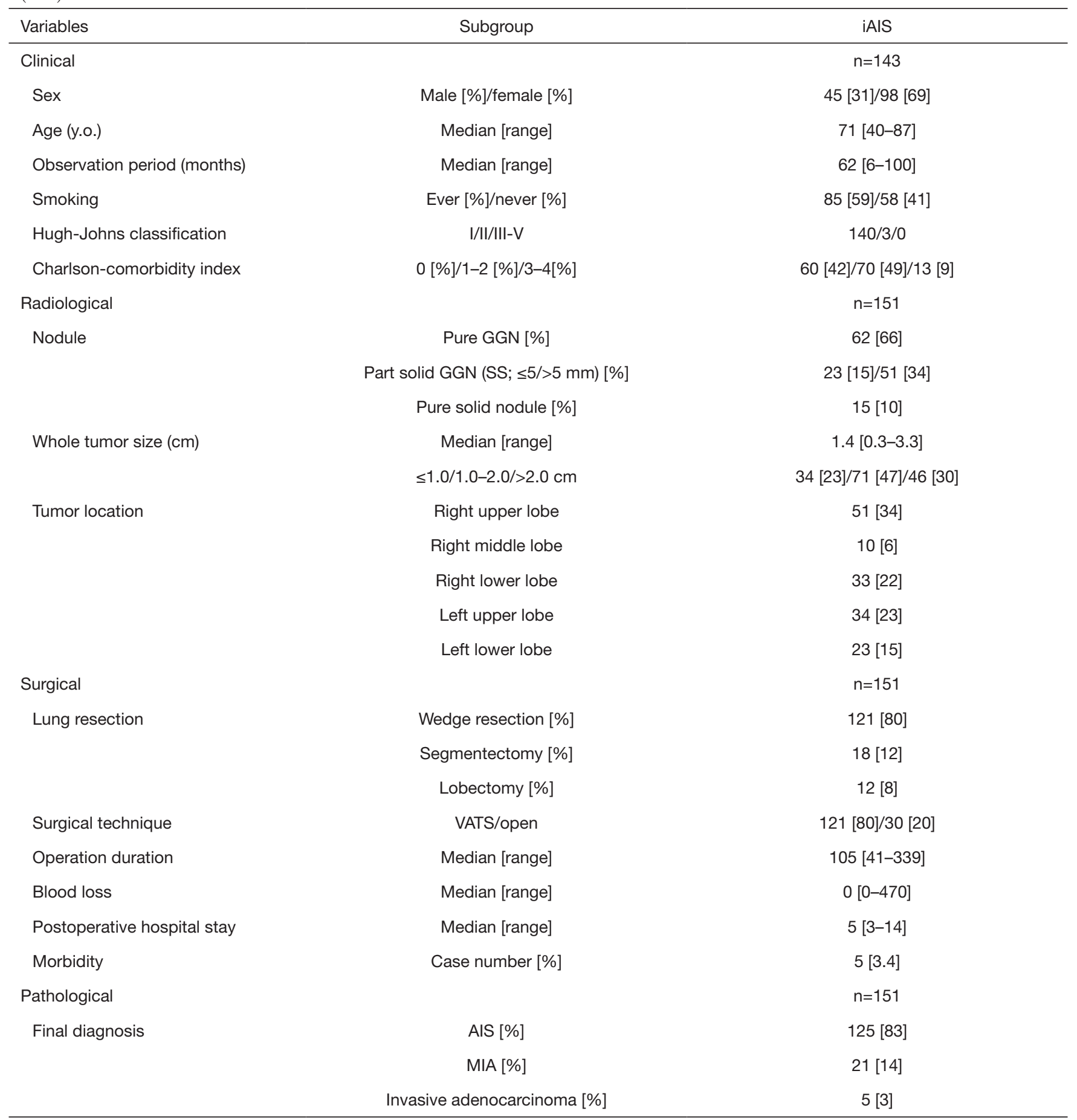

GGN, ground glass nodule; SS, solid component size; AIS, adenocarcinoma in situ; MIA, minimally invasive adenocarcinoma; VATS, video-assisted thoracic surgery. 
HRCT

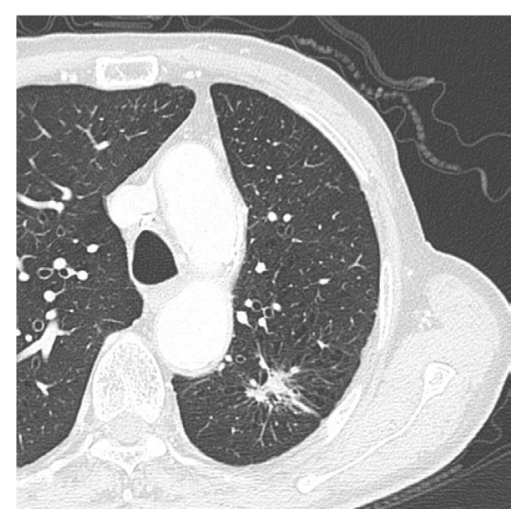

Frozen H.E.

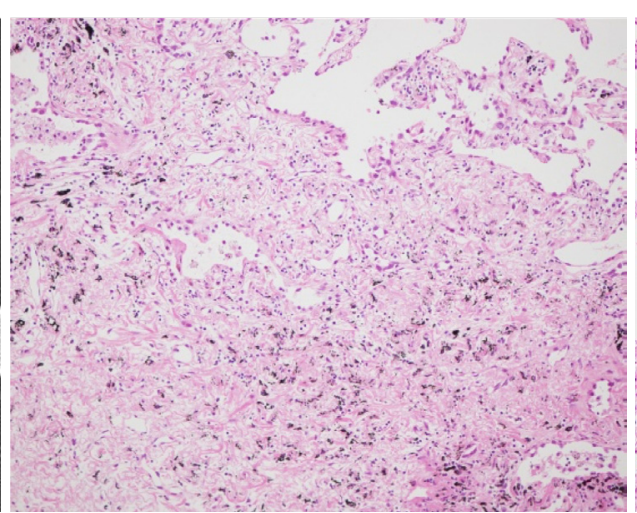

FFPE H.E.

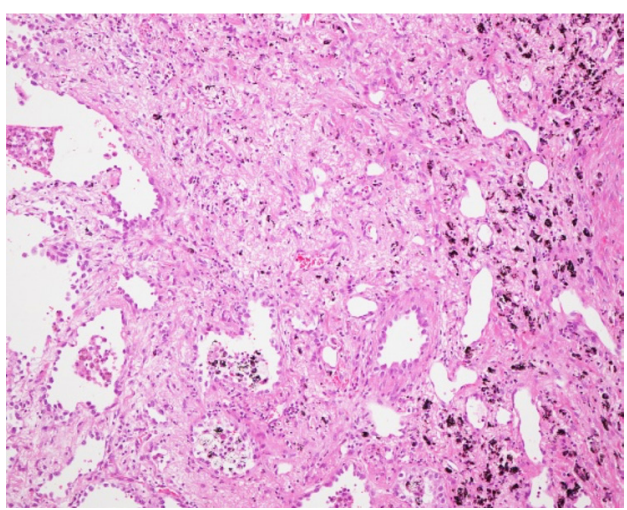

Figure 2 A typical case in which we could avoid excessive lobectomy. Images including high-resolution computed tomography and stained slides of a typical case. Although this nodule has no ground-glass component radiologically, the frozen-section diagnosis is AIS. The final pathological diagnosis is also AIS. The magnification is 200x. HRCT, high resolution computed tomography; HE, hematoxylin-eosin staining; FFPE, formalin-fixed paraffin-embedded; AIS, adenocarcinoma in situ.

Table 2 The breakdown list of surgical and pathological characteristics of 5 cases diagnosed as invasive adenocarcinoma

\begin{tabular}{lccc}
\hline Case & Lung resection & Predominant subtype & Invasive size $(\mathrm{cm}) /$ tumor size $(\mathrm{cm})$ \\
\hline 1 & Lobectomy & Lepidic predominant & $0.8 / 2.0$ \\
2 & Lobectomy & Lepidic predominant & $0.7 / 2.9$ \\
3 & Lobectomy & Lepidic predominant & $1.0 / 3.0$ \\
4 & Segmentectomy & Lepidic predominant & $0.8 / 1.3$ \\
5 & Wedge resection & Papillary predominant & $0.9 / 1.4$ \\
\hline
\end{tabular}

Therefore, it is vital to diagnose lung nodules in terms of tumor invasiveness intraoperatively when the patient undergoes limited resection. As preoperative radiological evaluation of lung cancer, generally, HRCT and positron emission tomography (PET) in addition to conventional $\mathrm{CT}$ are predominantly performed. In many GGN cases, PET evaluation is insufficient because the GGN is usually too small to provide precise information regarding tumor invasiveness. Accordingly, HRCT findings are much more reliable for their evaluation. However, even HRCT findings are sometimes confusing since some GGNs involve lung collapse, which looks like an invasive part. Unless intraoperative evaluation of GGN invasiveness is conducted, lobectomy could be performed for AIS with large lung collapse. Indeed, 15 radiologically pure-solid tumors were diagnosed as iAIS, of which 14 nodules (93.9\%) were confirmed as AIS. Surgeons should avoid excessive resection of the lung, and intraoperative evaluation of tumor cells with respect to their invasiveness is important.
In this study, the accuracy rate was high, $82.7 \%$. It is difficult to set a cut-off percentage for confirming this method as a clinically reliable one. However, as shown in Table 1 and Figure 2, cases of discordance cases did occur. Fortunately, tumor recurrence has not been identified in these cases. Liu and co-workers reported that the concordance rate between frozen section diagnosis and final pathology of peripheral lung adenocarcinoma was $84.4 \%$ (11). They also mentioned the accuracy rate of iAIS as in our study was $73.8 \%$. Walts et al. also demonstrated the concordance rate between intraoperative and final diagnosis of AIS was 59\% (12). Both reports revealed the accuracy rate depended on tumor size. Tumor size would be one of the reasons why the rate in our study is higher compared to these two studies. Of course, this rate should be $100 \%$. However, practically, it is not realistic when tumor size is very small and one slice of tumor was taken for frozen section diagnosis. Additionally, a GGN is often too soft to palpate, which makes it difficult for frozen slides to have 

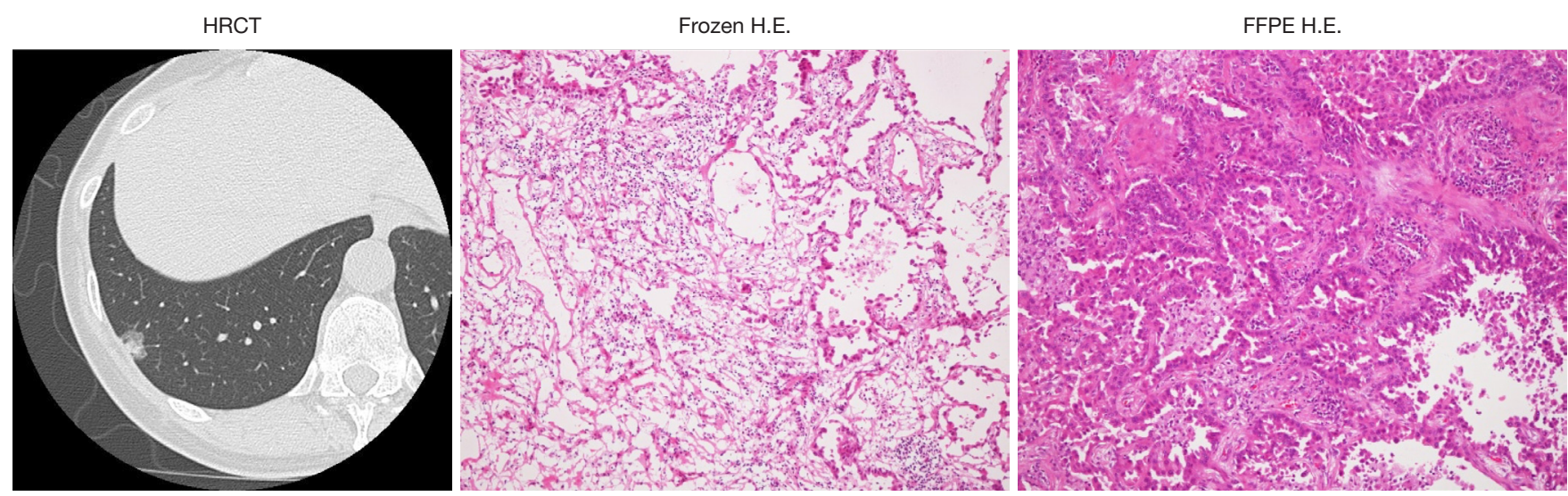

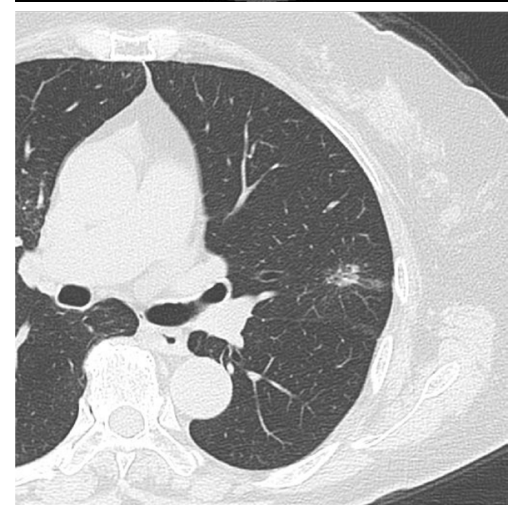

HRCT

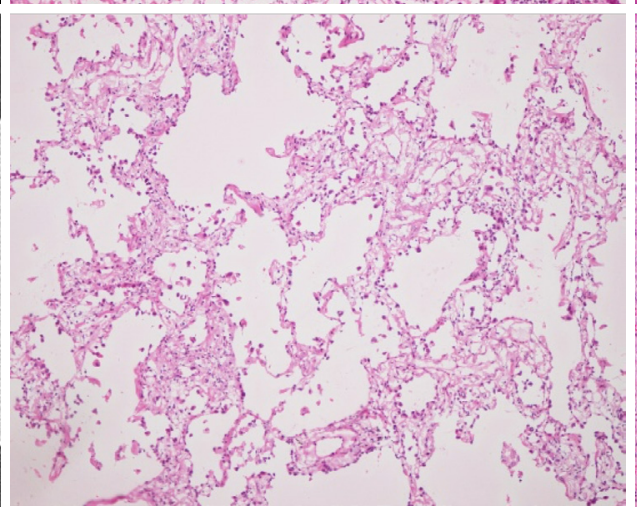

Frozen H.E.

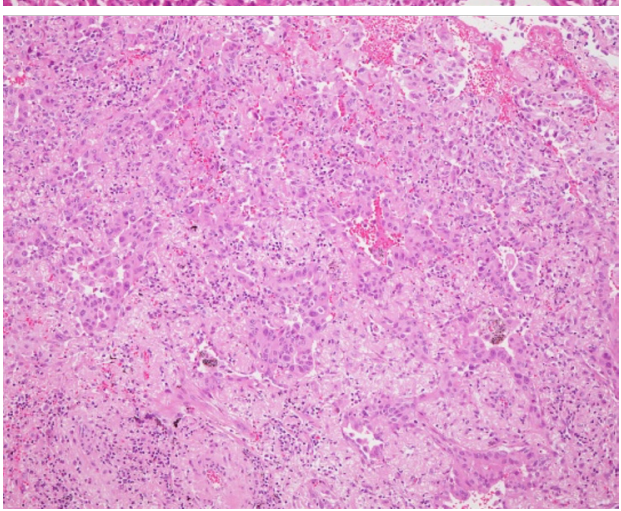

FFPE H.E.

Figure 3 Two discordant cases of adenocarcinoma in situ intraoperatively diagnosed as invasive adenocarcinoma postoperatively. Images of patients with adenocarcinoma in situ intraoperatively diagnosed as invasive adenocarcinoma postoperatively. In both cases, although frozensection slides do not reveal invasive features, such as destruction of the original fibrotic texture, formalin-fixed and paraffin-embedded slides show invasive components, such as papillary and acinar patterns, indicating invasive adenocarcinoma. The magnification is 200x. HRCT, high resolution computed tomography; HE, hematoxylin-eosin staining; FFPE, formalin-fixed paraffin-embedded.

Table 3 The breakdown list of intraoperative and final diagnosis of 497 intraoperatively-diagnosed adenocarcinoma, $\leq 3 \mathrm{~cm}$

\begin{tabular}{lccr}
\hline & \multicolumn{3}{c}{ Adenocarcinoma diagnosed intraoperatively, $\leq 3 \mathrm{~cm}(\mathrm{n}=497)$} \\
\cline { 2 - 4 } Final diagnosis & AIS $(\mathrm{n}=151)$ & MIA $(\mathrm{n}=40)$ & Invasive adenocarcinoma $(\mathrm{n}=306)$ \\
\hline AIS [\%] & $125[83]$ & $5[12.5]$ & $8[2]$ \\
MIA [\%] & $21[14]$ & $25[62.5]$ & $9[3]$ \\
Invasive adenocarcinoma [\%] & $5[3]$ & $10[25]$ & $289[95]$ \\
\hline
\end{tabular}

AIS, adenocarcinoma in situ; MIA, minimally invasive adenocarcinoma.

an accurate section level during a short time for precise evaluation of the depth of invasion. Pathologically, AIS is often accompanied by a solid alveolar wall and inflammatory hypertrophy of the lung tissue with wide fibrosis, which is difficult to distinguish from tumor stroma due to tumor invasion (13). Elastic fiber staining (e.g., Elastica Van Gieson staining) would be very useful to differentiate them. At our institute, if needed, intraoperative frozen-section diagnosis based on elastic fiber staining in addition to hematoxylineosin staining has been used not only for diagnosis of malignancy but also for evaluation of the extent of the invasive component for lung adenocarcinomas since 1998. 
Table 4 The breakdown list of radiological and pathological characteristics of 151 intraoperatively-diagnosed AIS

\begin{tabular}{llc}
\hline Radiological features & Final pathology diagnosis & Cases \\
\hline Pure GGN & AIS/MIA/invasive adenocarcinoma & $58 / 4 / 0$ \\
Part solid GGN $(\mathrm{SS} ; \leq 5 \mathrm{~mm})$ & AIS/MIA/invasive adenocarcinoma & $18 / 5 / 0$ \\
Part solid GGN $(\mathrm{SS} ;>5 \mathrm{~mm})$ & AIS/MIA/invasive adenocarcinoma & $35 / 12 / 4$ \\
Pure solid nodule & AIS/MIA/invasive adenocarcinoma & $14 / 0 / 1$ \\
\hline
\end{tabular}

AIS, adenocarcinoma in situ; GGN, ground glass nodule; SS, solid component size; AIS, adenocarcinoma in situ; MIA, minimally invasive adenocarcinoma.

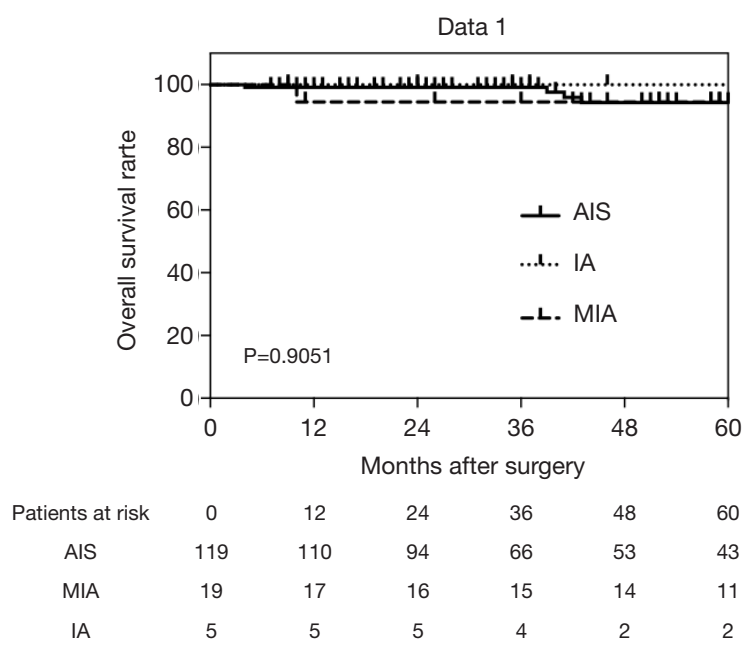

Figure 4 Overall survival after surgical resection diagnosed as adenocarcinoma in situ intraoperatively according to the final diagnosis. Overall survival curves classified by final diagnosis in patients diagnosed with AIS intraoperatively AIS. MIA, minimally invasive adenocarcinoma; IA, invasive adenocarcinoma; AIS, adenocarcinoma in situ.

As aforementioned, 21 patients with iAIS were finally diagnosed as having MIA. A few retrospective studies have demonstrated that limited resection is enough for MIA cases (14). If limited resection such as segmentectomy and wedge resection are revealed as a feasible operation for MIA, our evaluation is essential. In addition, although we did not find cases with discordance between MIA intraoperatively and AIS postoperatively, that may happen. Future research to reduce the discordance is required.

This study had some limitations. First, this study is retrospective. Second, for survival analysis, some cases need a longer observation period. Since adenocarcinoma with GGO is generally less invasive, even if tumor recurrence would occur due to incomplete resection for MIAs and invasive adenocarcinomas, it might occur more than in 5 years. Third, although consensus of radiological and pathological diagnosis was obtained among a couple of radiologists and pathologists respectively, this study was performed at one institute. In order to generalize our results, multicentral study should be required. Finally, this study enrolled pure GGNs, which would not be invasive adenocarcinoma. Further study including only solid-part GGNs and pure-solid nodules should be performed.

\section{Conclusions}

In conclusion, we achieved a high concordance rate of AIS between the diagnoses by intraoperative frozen sections and postoperative paraffin-embedded sections. Our intraoperative evaluation of frozen-section H.E. staining will be very useful for identifying tumor invasiveness of small-size lung adenocarcinomas and potentially avoiding excessive lung resection for lung AIS.

\section{Acknowledgments}

Funding: None.

\section{Footnote}

Reporting Checklist: The authors have completed the STARD reporting checklist. Available at http://dx.doi. org/10.21037/jtd-20-2710

Data Sharing Statement: Available at http://dx.doi. org/10.21037/jtd-20-2710

Peer Review File: Available at http://dx.doi.org/10.21037/jtd20-2710

Conflicts of Interest: All authors have completed the ICMJE 
uniform disclosure form (available at http://dx.doi. org/10.21037/jtd-20-2710). The authors have no conflicts of interest to declare.

Ethical Statement: The authors are accountable for all aspects of the work in ensuring that questions related to the accuracy or integrity of any part of the work are appropriately investigated and resolved. The study was conducted in accordance with the Declaration of Helsinki (as revised in 2013). This study was approved by the Institutional Review Board (IRB) of Tokyo Metropolitan Cancer and Infectious Diseases Center Komagome Hospital (IRB number 2481). The need for written informed consent was waived because of the retrospective study design.

Open Access Statement: This is an Open Access article distributed in accordance with the Creative Commons Attribution-NonCommercial-NoDerivs 4.0 International License (CC BY-NC-ND 4.0), which permits the noncommercial replication and distribution of the article with the strict proviso that no changes or edits are made and the original work is properly cited (including links to both the formal publication through the relevant DOI and the license). See: https://creativecommons.org/licenses/by-nc-nd/4.0/.

\section{References}

1. Oudkerk M, Devaraj A, Vliegenthart R, et al. European position statement on lung cancer screening. Lancet Oncol 2017;18:e754-66.

2. Yang D, Liu Y, Bai C, et al. Epidemiology of lung cancer and lung cancer screening programs in china and the united states. Cancer Lett 2020;468:82-7.

3. Sagawa M, Sugawara T, Ishibashi N, et al. Efficacy of lowdose computed tomography screening for lung cancer: The current state of evidence of mortality reduction. Surg Today 2017;47:783-88.

4. Patz EF Jr, Goodman PC, Bepler G. Screening for lung cancer. N Engl J Med 2000;343:1627-33.

5. Eguchi T, Kadota K, Park BJ, et al. The new iaslc-

Cite this article as: Shima T, Kinoshita T, Sasaki N, Uematsu M, Sugita Y, Shimizu R, Harada M, Hishima T, Yamamoto A, Horio H. Feasibility of intraoperative diagnosis of lung adenocarcinoma in situ to avoid excessive resection. J Thorac Dis 202 1;13(3):1338-1346. doi: 10.21037/jtd-20-2710 ats-ers lung adenocarcinoma classification: What the surgeon should know. Semin Thorac Cardiovasc Surg 2014;26:210-22.

6. Aokage K, Yoshida J, Hishida T, et al. Limited resection for early-stage non-small cell lung cancer as functionpreserving radical surgery: A review. Jpn J Clin Oncol 2017;47:7-11.

7. Goldstraw P, Chansky K, Crowley J, et al. The IASLC lung cancer staging project: Proposals for revision of the tnm stage groupings in the forthcoming (eighth) edition of the TNM classification for lung cancer. J Thorac Oncol 2016;11:39-51.

8. Travis WD, Asamura H, Bankier AA, et al. The IASLC Lung Cancer Staging Project: Proposals for coding T categories for subsolid nodules and assessment of tumor size in part-solid tumors in the forthcoming eighth edition of the TNM classification of lung cancer. J Thorac Oncol 2016;11:1204-23.

9. Tsutani Y, Miyata Y, Mimae T, et al. The prognostic role of pathologic invasive component size, excluding lepidic growth, in stage I lung adenocarcinoma. J Thorac Cardiovasc Surg 2013;146:580-5.

10. Sagawa M, Oizumi H, Suzuki H, et al. A prospective 5-year follow-up study after limited resection for lung cancer with ground-glass opacity. Eur J Cardiothorac Surg 2018;53:849-56.

11. Liu S, Wang R, Zhang Y, et al. Precise diagnosis of intraoperative frozen section is an effective method to guide resection strategy for peripheral small-size lung adenocarcinoma. J Clin Oncol 2016;34:307-13.

12. Walts AE, Marchevsky AM. Root cause analysis of problems in the frozen section diagnosis of in situ, minimally invasive, and invasive adenocarcinoma of the lung. Arch Pathol Lab Med 2012;136:1515-21.

13. Van Schil PE, Sihoe AD, Travis WD. Pathologic classification of adenocarcinoma of lung. J Surg Oncol 2013;108:320-6.

14. Yano M, Yoshida J, Koike T, et al. The outcomes of a limited resection for non-small cell lung cancer based on differences in pathology. World J Surg 2016;40:2688-97. 